\title{
Der ÖGD - Stark für die Schwachen, aber wie lange noch?
}

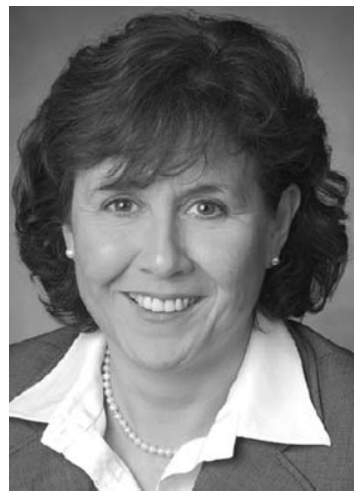

Dr. Ute Teichert-Barthel

\section{Bibliografie DOI http://dx.doi.org/ 10.1055/s-0033-1343422 Gesundheitswesen 2013; 75: $177-178$ (c) Georg Thieme Verlag KG Stuttgart · New York ISSN 0941-3790}

Korrespondenzadresse
Dr. Ute Teichert-Barthel
Vorsitzende BVÖGD
Bundesgeschäftstelle
c/o Gesundheitsamt Charlot-
tenburg-Wilmersdorf
Hohenzollerndamm 174
10713 Berlin
ute.teichert@uminfo.de

Dr. Ute Teichert-Barthel Vorsitzende BVÖGD Bundesgeschäftstelle c/o Gesundheitsamt Charlot(asdorf 10713 Berlin ute.teichert@uminfo.de
Liebe Kolleginnen und Kollegen,

Sie besuchen in diesen Tagen den 63. Wissenschaftlichen Kongress der Bundesverbände der Ärztinnen und Ärzte sowie der Zahnärzte des Öffentlichen Gesundheitsdienstes. Der Kongress steht unter dem Motto „Der Öffentliche Gesundheitsdienst - Stark für die Schwachen“.

Dieser wissenschaftliche Kongress wird jährlich an wechselnden Orten bundesweit veranstaltet. In diesem Jahr ist der Landesverband Brandenburg-Berlin für die Ausrichtung zuständig. An 3 Tagen haben Sie die Möglichkeit, sich in bis zu 8 parallelen Veranstaltungen fachlich auszutauschen und neueste Erkenntnisse aus Wissenschaft und Praxis vermittelt zu bekommen. Das weitere vielfältige Kongressangebot zu Vorträgen, Workshops, Präsentationen sowie kulturellen und touristischen Highlights können Sie in allen Einzelheiten dem fast 100 Seiten umfassenden Programmheft entnehmen.

In der vorliegenden Ausgabe des Gesundheitswesen finden Sie ausgewählte Vorträge vom letztjährigen Kongress in Erfurt, die in einer längeren Fassung veröffentlicht werden. Damit wollen wir die Möglichkeit nutzen, das breite Aufgabenspektrum und die fachlich hoch qualifizierten Arbeiten im ÖGD einem breiteren Publikum zugängig zu machen. Der öffentliche Gesundheitsdienst (ÖGD) hat ganz zu Unrecht bei vielen Ärzten ein schlechtes Image, dem wollen wir als Berufsverband entgegenwirken.

Wichtig ist auch, dass wir unsere Fachexpertise in die aktuelle politische Diskussion mit einbringen. So wurde Ende März der Entwurf eines „Gesetzes zur Förderung der Prävention“ vom Bundeskabinett beschlossen. Das ist für den ÖGD ein ganz wichtiger Tätigkeitsbereich, hier haben wir die Chance, wirklich etwas zu bewegen. Die Präventionsarbeit vor Ort ist sehr stark an den Gesundheitsämtern angesiedelt.

Es gibt im Präventionsbereich hervorragende Initiativen, die man bereits mit wenig Geld und wenig Aufwand umsetzen könnte. Dem Stellenwert des ÖGD im Bereich der Prävention wird bislang in dem Gesetzentwurf, der sich fast ausschließlich auf Veränderungen im SGB V beschränkt, nicht ausreichend Rechnung getragen. Hier besteht im weiteren parlamentarischen Beratungsverfahren noch erheblicher Nachholbedarf.

Mit Blick auf die Einbindung des ÖGD werden wertvolle Chancen, aber auch spezifisches knowhow und Ressourcen bislang nicht genutzt. Die im Gesetzentwurf zusätzlich vorgesehenen Finanzmittel für Verbesserungen bei präventiven Maßnahmen sind leider nicht unmittelbar für den ÖGD vorgesehen. Hier sollten wir gemein- sam darauf hinwirken, unsere spezifischen Interessen und Angebote gegenüber den Akteuren und Kooperationspartnern offensiv zu vertreten. Nur wenn zukünftig alle Akteure in der Prävention vernetzt, zielgruppenspezifisch und gemeinsam agieren, lässt sich das Erreichte weiter ausbauen.

Neben der Prävention wird das Thema Krankenhaushygiene in den letzten Jahren in den Medien und der Politik intensiv diskutiert. Mit zahlreichen Gesetzen auf Bundes- und Landesebene versucht man strukturelle und personelle Verbesserungen in den Krankenhäusern zu etablieren. Davon ist auch der ÖGD betroffen, denn die infektionshygienische Überwachung von Gesundheitseinrichtungen wie Kliniken, Altenheimen und Arztpraxen liegt bei den Gesundheitsämtern.

In den Landesverordnungen wird nach Vorgaben der KRINKO flächendeckend konkret geregelt, wie viele Krankenhaushygieniker, hygienebeauftragte Ärzte und Hygienefachkräfte pro Bettenzahl beschäftigt werden sollen. Unstrittig ist, dass in den Einrichtungen der personelle Bedarf im ärztlichen und pflegerischen Bereich für die Krankenhaushygiene aufgestockt werden muss, wenn man Verbesserungen erreichen will. Nach den aktuellsten Plänen der Regierungskoalition sollen den Krankenhäusern hierfür in den nächsten Jahren die Finanzierung zusätzlicher Stellen ermöglicht werden.

Nur, wie soll mit diesen Mitteln die infektionshygienische Überwachung durch die Gesundheitsämter sicher gestellt werden? Auch hier ist eine personelle Verstärkung notwendig. Aus unserer Sicht sollte daher der Fokus der Personalaufstockung nicht nur auf den Krankenhausbereich, sondern auch auf die hygieneüberwachenden Behörden gelegt werden.

Neben den Vorkommnissen in den Neonatologiestationen Bremen und Mainz hat auch die EHEC Krise gezeigt, wie wichtig eine adäquate personelle Ausstattung in den Gesundheitsämtern ist. Nicht nur die Krankenhäuser haben ein Organisationsverschulden bei zu wenig Hygienepersonal zu rechtfertigen. Auch die politischen Verantwortlichen sind in der Pflicht, im Sinne der Daseinsfürsorge für die Bürgerinnen und Bürger bundesweit die Gesundheitsämter ausreichend finanziell und personell auszustatten.

In diesem Zusammenhang ist auch ein weiteres großes Problem zu nennen, mit dem der ÖGD aktuell zu kämpfen hat, der Nachwuchsmangel. Schon jetzt arbeiten insgesamt weniger als $1 \%$ der Beschäftigten im Gesundheitswesen im 
Öffentlichen Gesundheitsdienst. Die Gesamtzahl der Ärztinnen und Ärzte im Öffentlichen Gesundheitsdienst beläuft sich auf ca. 2800, davon sind rund 800 Fachärztinnen und Fachärzte in den Gesundheitsämtern tätig. Sie sehen, wir sind nur eine sehr kleine Gruppe und unsere Zahl ist weiter rückläufig.

Nach einer aktuellen Umfrage unseres Berufsverbandes zur Stellensituation in den Gesundheitsämtern waren im letzten Jahr rund 200 Stellen länger als 6 Monate nicht besetzt. Hier sind alle aufgerufen, aktiv zu werden. Setzen Sie sich vor Ort dafür ein, dass frei werdende Stellen unverzüglich nachbesetzt werden. Machen Sie die politisch Verantwortlichen darauf aufmerksam, dass im Sinne einer regionale Gesundheitsversorgung für alle Bürgerinnen und Bürger der ÖGD als dritte Säule im Gesund- heitswesen adäquat finanziell und personell auszustatten ist. Nicht nur der hausärztliche Bereich hat erhebliche Versorgungsprobleme, auch im ÖGD drohen - nicht zuletzt vor dem Hintergrund einer ungünstigen Altersstruktur - zunehmende Engpässe. Nur wenn wir die Verantwortlichen vor Ort sensibilisieren und in die Verantwortung nehmen, wird es gelingen, auch auf höherer Ebene ein Umdenken zu erreichen. Vielleicht bewegt sich dann auch etwas im Tarifbereich auf allen Ebenen.

Mit freundlichen kollegialen Grüßen

Ihre

Dr. Ute Teichert-Barthel 